\title{
SUMO1/UBC9-decreased Nox1 activity inhibits reactive oxygen species generation and apoptosis in diabetic retinopathy
}

\author{
JIAOLI HU*, PENGCHENG XUE* , XINBANG MAO, LIN XIE, GUODONG LI and ZHIPENG YOU \\ Department of Ophthalmology, The Second Affiliated Hospital of Nanchang University, Nanchang, Jiangxi 330006, P.R. China
}

Received February 4, 2017; Accepted August 14, 2017

DOI: $10.3892 / \mathrm{mmr} .2017 .8037$

\begin{abstract}
Diabetic retinopathy (DR) is an increasing global health concern that causes vision loss and blindness. Reactive oxygen species (ROS) are considered to be a principal cause of DR. An important source of ROS is the oxidization of NADPH. In the present study, NADPH oxidase 1 (Nox1)-expressing human retinal epithelial cell (HREC) lines were generated and infected with small ubiquitin-like modifier 1 (SUMO1) and/or ubiquitin conjugating enzyme E2 I (UBC9) lentiviral pGMLV constructs. The viabilities, apoptotic capacities and ROS production levels of the HREC lines were quantified using Hoechst 33258, annexin V/propidium iodide and dichlorodihydrofluorescein diacetate assays, respectively. Additionally, rat DR models were established. From these models, the apoptotic capacities of retinal tissues were visualized using terminal deoxynucleotidyl transferase dUTP nick end labeling assays, and the pathologies were evaluated. The mRNA and protein expression levels of SUMO1, UBC9 and Nox1 were analyzed using reverse transcription-quantitative polymerase chain reaction and western blot analyses, respectively. Compared with controls, the relative mRNA levels of SUMO1 and UBC9 were significantly upregulated, and the Nox1 levels significantly downregulated, in cells infected with SUMO1 or UBC9 alone or in combination. The ROS production and apoptosis rates of cells and retinal tissues were decreased. In addition, pathological symptoms in DR tissues improved when they were simultaneously transfected with SUMO1 and UBC9 via intraocular injection. In conclusion, the SUMO1/UBC9 axis may regulate Nox1-mediated DR by inhibiting ROS generation and apoptosis in rat and cellular model systems.
\end{abstract}

Correspondence to: Dr Zhipeng You, Department of Ophthalmology, The Second Affiliated Hospital of Nanchang University, 1 Minde Road, Nanchang, Jiangxi 330006, P.R. China E-mail: yzpengy@163.com

${ }^{*}$ Contributed equally

Key words: small ubiquitin-like modifier, NADPH oxidase, reactive oxygen species, diabetic retinopathy, apoptosis

\section{Introduction}

Diabetic retinopathy (DR) is a major global cause of vision loss and blindness. It was reported that, of $28.5 \%$ (4.2 million) cases of blindness reported between 2005 and 2008 in the USA, $>40 \%$ were able to be attributed to DR (1). The most well-known risk factors for DR progression are the length of time with diabetes (hyperglycemia) and inadequate glycemic control (2). Patients with 5-year medical histories of diabetes mellitus had a $\sim 17 \%$ chance of developing DR; patients who were diabetic for at least 15 years may have a $~ 97.5 \%$ chance of developing the disease (3). The classical features of DR include: Thickened basement membranes of retinal capillaries; permeability increases in retinal blood vessels; ocular tissue ischemia; and aberrant angiogenesis (4). Based on disease progression, DR may be classified as either non-proliferative (DR characterized by microaneurysms and small dilatations in retinal blood vessels), or proliferative (DR characterized by angiogenesis from the retinal surface, frequently leading to vitreous hemorrhages and retinal detachment) $(4,5)$.

Oxidative stress is the leading molecular cause of DR and is associated with the generation of the reactive oxygen species (ROS) $\mathrm{O}_{2}{ }^{-}$and $\mathrm{H}_{2} \mathrm{O}_{2}$. ROS molecules regulate endothelial function and vascular tone; excessive ROS may lead to cellular injuries (6). NADPH oxidase generates superoxide from oxygen, has been demonstrated to be an intracellular ROS source, and regulates the function of the vascular system (7-9). NADPH oxidase 1 (Nox1), is one of seven isoforms, in addition to Nox2-5 and dual oxidase 1-2. Nox1 was reported to be the predominant Nox isoform in retinal ganglion cells, compared with expression levels of Nox2 and Nox4 (10). Additionally, small ubiquitin-like modifier (SUMO) proteins are an evolutionarily conserved family of gene products that have been identified in all eukaryotes. Although SUMO proteins are structurally similar to ubiquitin, the two families do not share biological functions. Ubiquitination facilitates degradation of target proteins, while SUMOylation regulates a range of signal transduction and post-translational regulatory functions (11-13). SUMO1 protein, a member of the SUMO gene family, is subcellularly located in nuclear pore complexes and within nuclei.

As demonstrated in a number of previous studies, the SUMO1/ubiquitin conjugating enzyme E2 I (UBC9) pathway increased ROS production by regulating Nox gene expression levels in human embryonic kidney cells, human vascular 
smooth muscle cells and cancer cells $(14,15)$. Increased expression of Nox 1 caused excessive ROS and disorganized cellular functioning in pathological human case studies (16), and increased ROS-evoked aberrant SUMOylation via SUMO1 modification (17). In addition, SUMO family proteins and ROS overproduction have been implicated in the etiology of diabetes $(18,19)$. Despite a body of literature, associations between SUMO1/UBC9, ROS, apoptosis and Nox1 have not been elucidated. Additionally, the mechanistic underpinnings of SUMO1/UBC- and Nox1-mediated regulation of DR remain poorly understood.

The present study employed human retinal microvascular endothelial cell (HREC) lines to investigate whether SUMO1/UBC9 gene products may regulate Nox1-induced ROS and apoptosis in DR, using cell transfection, flow cytometry, and reverse transcription-quantitative polymerase chain reaction (RT-qPCR) methodologies in in vitro and in vivo model systems.

\section{Materials and methods}

Cell culture techniques and cell lines. HREC lines were purchased from a preservation commission cell bank (Chinese Academy of Sciences, Beijing, China). Cell culture and transfection techniques were performed as previously described $(20,21)$. The cells were cultured in extracellular matrix supplemented with 5\% fetal bovine serum (Gibco; Thermo Fisher Scientific, Inc., Waltham, MA, USA), endothelial cell growth supplement $(100 \mathrm{U} / \mathrm{ml})$ and $1 \%$ penicillin $/$ streptomycin at $37^{\circ} \mathrm{C}$ with $5 \% \mathrm{CO}_{2}$ for $48 \mathrm{~h}$. HREC cells stably expressing Nox1 were generated in the laboratory (22). Nox1-expressing HRECs (grown to 50-70\% confluence) were transfected with lentiviral vectors containing control, SUMO1, and/or UBC9 mRNA (30 nmol/l, Ambion) at $37^{\circ} \mathrm{C}$ in a $5 \% \mathrm{CO}_{2}$ incubator, and were incubated for a 48 -h period. The HRECs were divided into five groups: i) Nox1-expressing HRECs; ii) Nox1-expressing HRECs treated with $30 \mathrm{mM}$ glucose; iii) Nox1-expressing HRECs treated with $30 \mathrm{mM}$ glucose and a lentivirus containing SUMO1 mRNA; iv) Nox1-expressing HRECs treated with $30 \mathrm{mM}$ glucose and a lentivirus containing UBC9 mRNA; or v) Nox1-expressing HRECs treated with $30 \mathrm{mM}$ glucose and a lentivirus containing SUMO1 and UBC9 mRNA.

Lentiviral transfections. Full-length SUMO1 and UBC9 cDNA was amplified with primers against the NheI and BamHI restriction digest sites using KOD-PLUS Neo polymerase at $37^{\circ} \mathrm{C}$ (Toyobo Life Science, Osaka, Japan). Amplified cDNA products were cloned into pGMLV lentiviral vectors (Shanghai GeneChem Co., Ltd., Shanghai, China) using NheI and BamHI restriction enzymes and DNA ligase. The recombinant vectors were combined with vesicular stomatitis virus, Rev and Gag vectors and the mixture was transfected into 293T cells using Lipofectamine 2,000 (Invitrogen; Thermo Fisher Scientific, Inc.) to obtain viral supernatants. When Nox1-expressing HRECs reached 50-70\% confluence, pGMLV lentiviral vectors overexpressing SUMO1 and/or UBC9 [multiplicity of infection (MOI) of 100 viral particles/cell] were added, with a polybrene supplement for infections. A total of $6 \mathrm{~h}$ post-infection, the culture medium was changed to Dulbecco's modified Eagle's medium (Gibco; Thermo Fisher Scientific, Inc.) supplemented with $10 \%$ serum for $48 \mathrm{~h}$ in standard conditions. The transfected cells were cultured with puromycin and SUMO1 and/or UBC9 expressions were verified using RT-qPCR analysis.

$R T-q P C R$ analysis. Total RNA was extracted from each HREC population using TRIzol reagent (Invitrogen, USA), according to the manufacturer's protocol. Using standard reverse transcription kit HiFiScript cDNA (CW2569; CWBIO, Beijing, China), $2 \mu \mathrm{g}$ total RNA was transcribed into cDNA. The RT-qPCR was performed on a StepOne Real-time PCR system (Applied Biosystem; Thermo Fisher Scientific, Inc.) using the following protocol: i) $70^{\circ} \mathrm{C}$ for $5 \mathrm{~min}$; ii) $37^{\circ} \mathrm{C}$ for $1 \mathrm{~h}$; and iii) $85^{\circ} \mathrm{C}$ for $10 \mathrm{~min}$. SUMO1, UBC9, Nox 1 and $\beta$-actin primers were synthesized by Sangon Biotech Co., Ltd. (Shanghai, China) and the sequences were: SUMO1 forward, 5'-ATTGCCCTTCTTCCTTTA-3' and reverse, 5'-TTCCAC AGTTCGGTTCTC-3'; Nox1 forward, 5'-CTGGGTGGTTAA CCACTGGTTT-3' and reverse, 5'-ACCAATGCCCGTGAA TCCCTAAG-3'; UBC9 forward, 5'-TCCTGATGGGACGAT GAACC-3' and reverse, 5'-TGGGTGGTGAAGAGGGGT AGT-3'; and $\beta$-actin forward, 5'-CCTCTATGCCAACACAGT GC-3' and reverse, 5'-GTACTCCTGCTTGCTGATCC-3'. The reference gene was $\beta$-actin. RT-qPCR was performed using SYBR Green qPCR SuperMix (Invitrogen; Thermo Fisher Scientific, Inc.), according to the manufacturer's protocol. The protocol for qPCR amplification was: i) $95^{\circ} \mathrm{C}$ for $55 \mathrm{sec}$; ii) 40 cycles of $95^{\circ} \mathrm{C}$ for $15 \mathrm{sec}$ and $60^{\circ} \mathrm{C}$ for $20 \mathrm{sec}$; iii) $72^{\circ} \mathrm{C}$ for $30 \mathrm{sec}$. The relative expression of the target genes was analyzed using the $2^{-\Delta \Delta \mathrm{Cq}}$ method (23).

Hoechst 33258 assay. The viabilities of the HREC lines were detected using Hoechst 33258 as described previously with minor modifications (24). The cells were fixed by treating with an acetic acid-ethanol solution, followed by a 5-min wash with sterile 0.01 M PBS. Subsequently, cells were stained with a working solution of Hoechst 33258 according to the manufacturer's instructions (Beyotime Institute of Biotechnology, Haimen, China) for $15 \mathrm{~min}$ at room temperature, followed by three 5-min washes with sterile 0.01 M PBS. Slides were mounted using a (1:9) mixture of glycerol and PBS. As Hoechst 33258 fluorescently stains the nuclear chromosomes of apoptotic cells, a fluorescence microscope (magnification, x200; Leica Microsystems GmbH, Wetzlar, Germany) was used to quantify the number of apoptotic cells. Initial concentrations of HRECs were $1.5 \times 10^{4}$ cells/well. Normal cells were counted in five randomly chosen fields to calculate the percentages of living cells in each cohort.

Annexin V/propidium iodide assay. HREC apoptosis levels were assessed using a KGA107 staining kit (Nanjing KeyGen Biotech Co., Ltd., Nanjing, China). Following EDTA-trypsin digestion, cells were pelleted by centrifugation at $450 \mathrm{xg}$ for $5 \mathrm{~min}$ at room temperature, rinsed twice in sterile PBS and resuspended in $500 \mu \mathrm{l}$ binding buffer. The cells were mixed with $5 \mu \mathrm{l}$ annexin V-fluorescein isothiocyanate and $5 \mu \mathrm{l}$ propidium iodide for 5-15 min of incubation at room temperature in the dark. Apoptotic events were observed and recorded on a FACScan flow cytometer (BD Biosciences, Frank lin Lakes, NJ, USA) equipped with FACSComp software (Quadra 650; both from BD Biosciences, Franklin Lakes, NJ, USA) within $1 \mathrm{~h}$. 


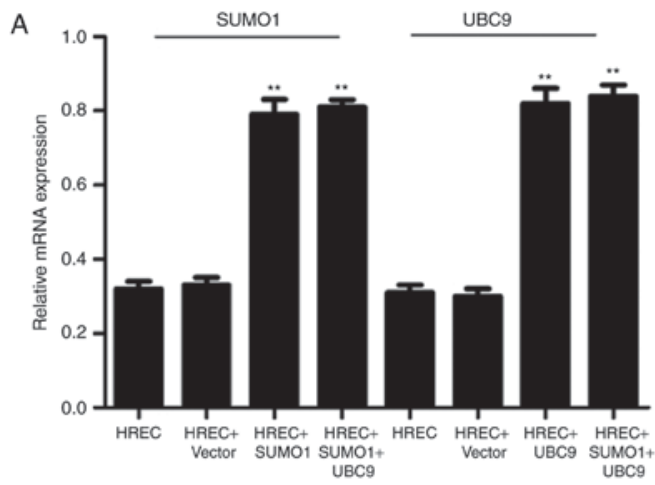

B
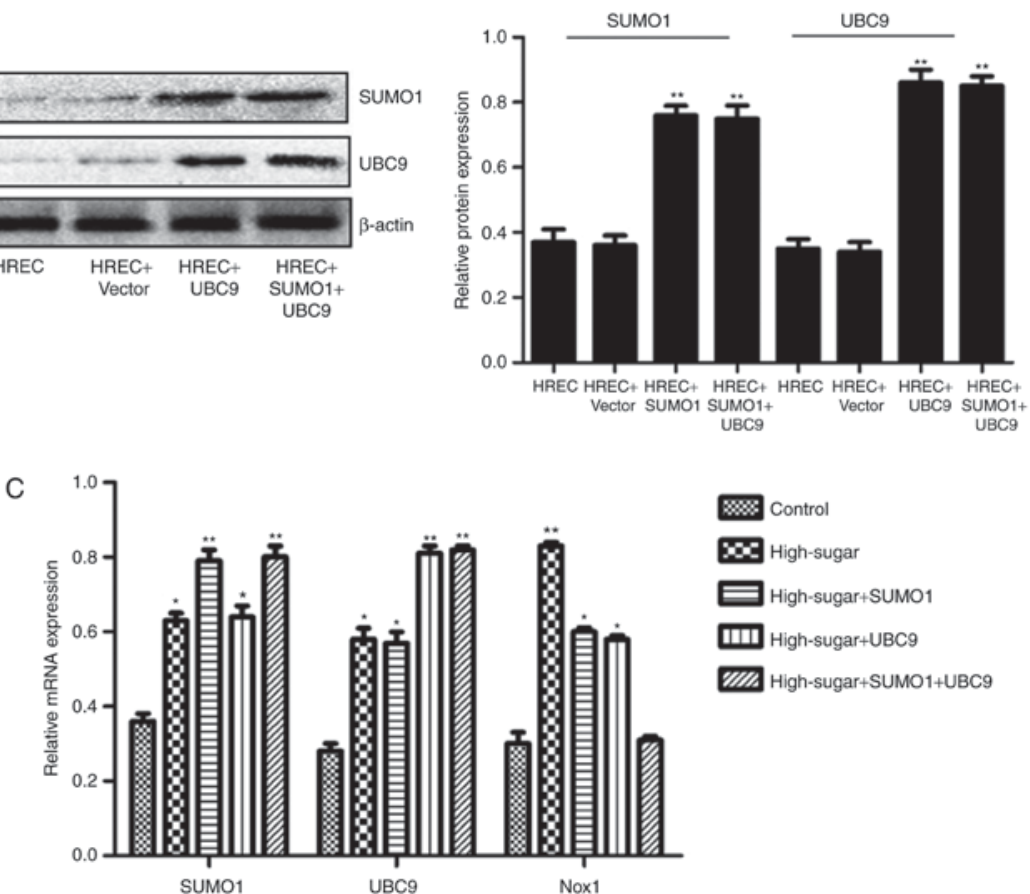

Figure 1. Constructions of SUMO1 and UBC9 lentiviral transfections and their effects on the expressions of SUMO1, UBC9 and Nox1 in Nox-1-expressing HRECs with high-glucose treatment. (A) Relative mRNA expression of SUMOI and UBC9. (B) Representative images of western blot analysis with the relative protein expression of SUMO1 and UBC9 in Noxl-expressing HRECs, post-lentiviral transfection with SUMO1 and/or UBC9. (C) Relative mRNA expression of SUMO1, UBC9 and Noxl in Nox-1-expressing HRECs following treatment with glucose $(30 \mathrm{mM})$ and lentiviral transfection with SUMO1, UBC9 or SUMO1 + UBC9. ${ }^{*} \mathrm{P}<0.05,{ }^{* *} \mathrm{P}<0.01$ vs. control. SUMO1, small ubiquitin-like modifier 1; UBC9, ubiquitin conjugating enzyme E2 I; Nox1, NADPH oxidase 1; HREC, human retinal microvascular endothelial cell; RT-qPCR, reverse transcription-quantitative polymerase chain reaction.

Intracellular ROS quantification assays. Intracellular ROS levels were evaluated by measuring the oxidative conversion of cell permeable dichlorodihydrofluorescein diacetate (DCFH-DA; Beyotime Institute of Biotechnology) to green fluorescent 2',7'-dichlorofluorescein (DCF;). DCFH-DA was dissolved in dimethyl sulfoxide to a final concentration of $5 \mu \mathrm{M}$. HREC lines $\left(1 \times 10^{6}\right.$ cells $\left./ \mathrm{ml}\right)$ were incubated with $2 \mathrm{mM}$ DCFH-DA for $20 \mathrm{~min}$ in standard conditions, followed by three washes in PBS and resuspension. The cells were assessed by flow cytometry (FACSCan; BD Biosciences) equipped with FACSComp software (Quadra 650), at excitation/emission wavelengths of $488 / 525 \mathrm{~nm}$.

Animals. Sprague-Dawley rats $(\mathrm{n}=25$; male; age, 4 years; weight, 180-220 g) were purchased from the Shanghai Laboratory Animal Center of the Chinese Academy of Sciences (Shanghai, China). The rats were maintained in facilities with controlled temperature $\left(23 \pm 2^{\circ} \mathrm{C}\right)$, humidity $(50 \%)$ and lighting (12 h light/12 h dark). The rats were fed a standard laboratory diet and had ad libitum access to tap water. All animals were treated humanely, in compliance with institutional animal care guidelines, and their used was approved by the Experimental Animal Ethics Committee of The Second Affiliated Hospital of Nanchang University (Nanchang, China). Animals were randomly divided into five groups: Healthy controls; DR; DR infected with a lentivirus containing SUMO1 mRNA; DR infected with a lentivirus containing UBC9 mRNA; and DR infected with a lentivirus containing both SUMO1 and UBC9 mRNA.

Establishment of diabetic retinopathy model. Before experiments were performed, streptozotocin (STZ; Sigma-Aldrich; Merck KGaA, Darmstadt, Germany), which has been frequently used in the establishment of DR models (25), was dissolved in $0.1 \mathrm{M}$ citrate buffer ( $\mathrm{pH} 4.5$ ). Rats were anesthetized with $1 \%$ pentobarbital sodium $[40 \mathrm{mg} / \mathrm{kg}$ injected intraperitoneally (i.p.)] and received a $65 \mathrm{mg} / \mathrm{kg}$ i.p. injection of STZ. Rats in the healthy control group received an 

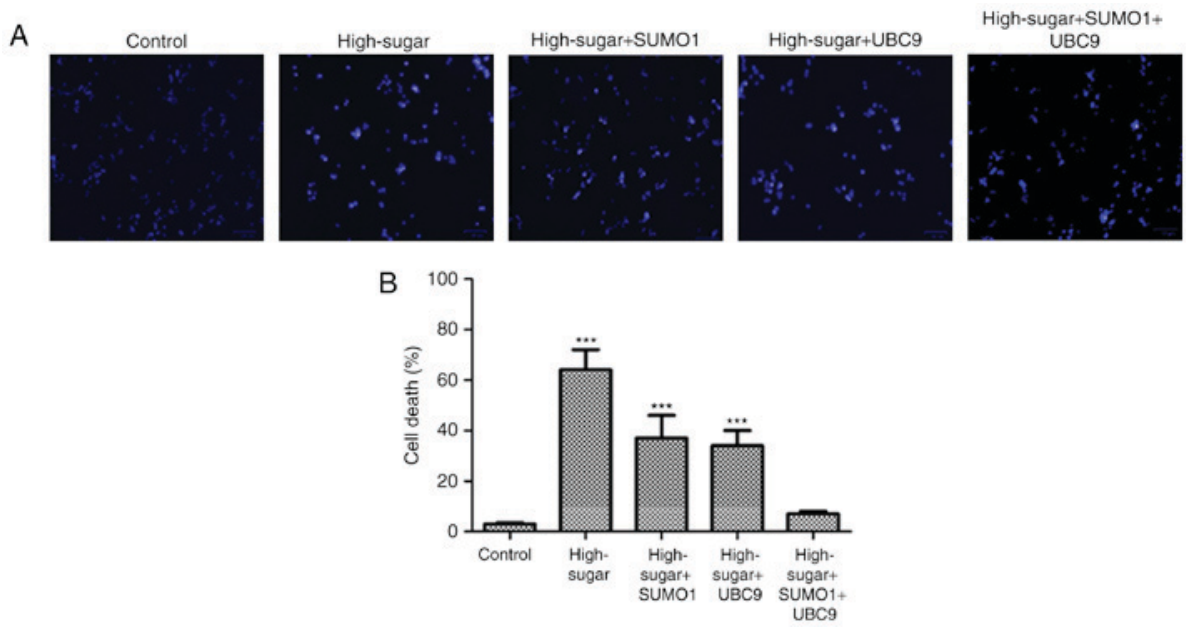

Figure 2. Evaluation of HREC cells by Hoechst 33258. (A) Morphology of apoptotic HRECs using Hoechst 33258 staining (x200) and (B) cell death rates of HRECs were assessed. HRECs were divided in five groups: Nox1-expressing controls (control), Nox1-expressing cells treated with 30 mM glucose (high sugar), Noxl-expressing cells treated with $30 \mathrm{mM}$ glucose and transfected with SUMO1 (high-sugar + SUMO1), Noxl-expressing cells treated with 30 mM glucose and transfected with UBC9 (high-sugar + UBC9), and Noxl-expressing cells treated with $30 \mathrm{mM}$ glucose and transfected with SUMO1 and UBC9 (high-sugar + SUMO1 + UBC9). ${ }^{* * *} \mathrm{P}<0.001$ vs. control. HREC, human retinal microvascular endothelial cell; SUMO1, small ubiquitin-like modifier 1; UBC9, ubiquitin conjugating enzyme E2 I; Nox1, NADPH oxidase 1.
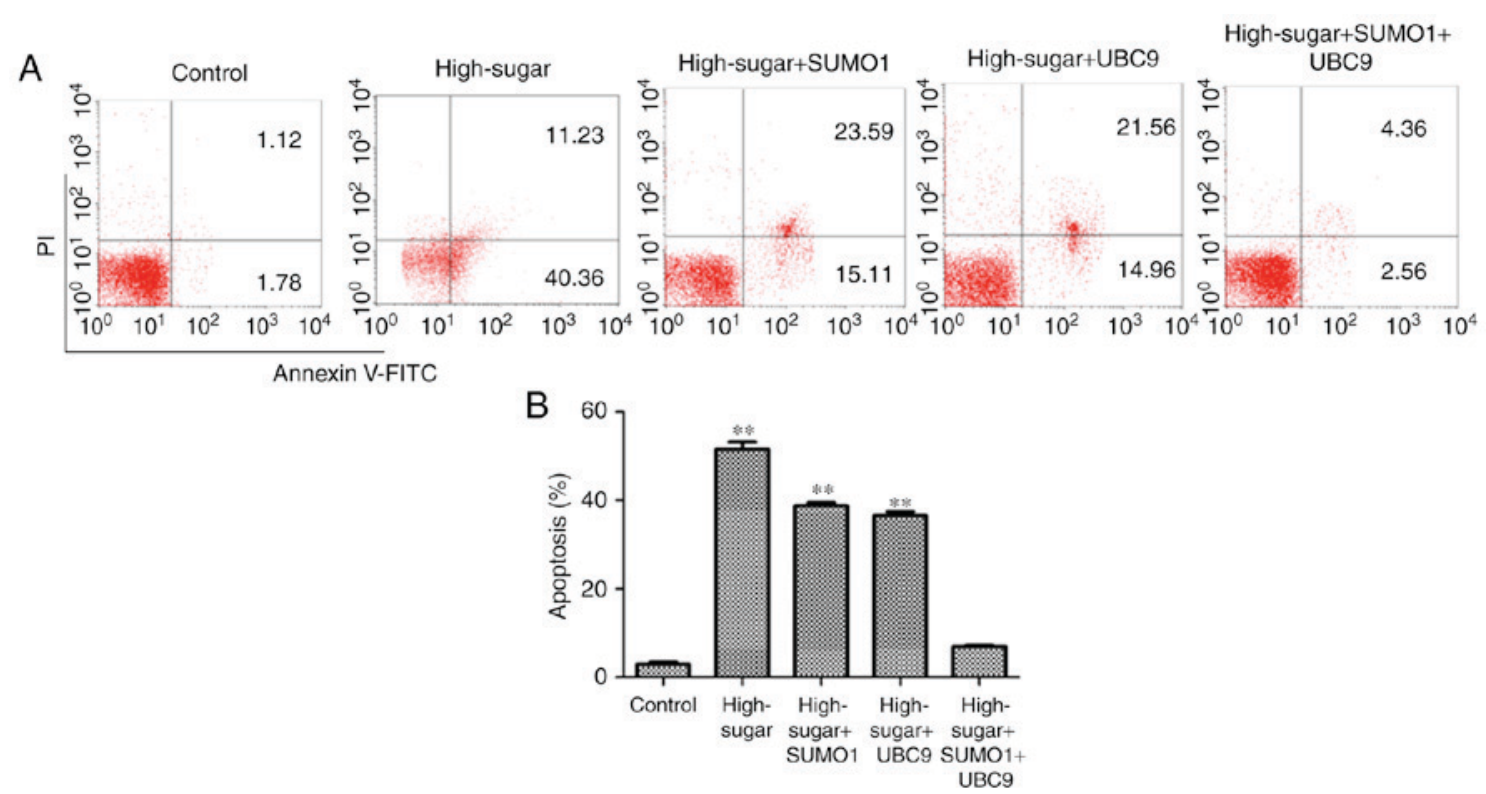

Figure 3. Evaluation of HREC cells by flow cytometry. Apoptosis (A) analysis and (B) quantification of HRECs across the five treatment groups. ${ }^{* *} \mathrm{P}<0.01$ vs. controls. PI, propidium iodide; FITC, fluorescein isothiocyanate; SUMO1, small ubiquitin-like modifier 1; UBC9, ubiquitin conjugating enzyme E2 I.

equivalent dose of the citrate buffer vehicle. Following $72 \mathrm{~h}$ of incubation, glucose levels were monitored using blood glucose meters (One Touch UltraEasy; Lifescan, Inc., Milpitas, CA, USA); animals with blood glucose levels $>16.7 \mathrm{mM}$ were considered to be diabetic. Subsequently, $20 \mu 1$ appropriate lentiviral mixtures were injected into the vitreous cavities of both eyes. The mice were sacrificed with $1 \%$ pentobarbital sodium ( $80 \mathrm{mg} / \mathrm{kg}$ i.p.), and the eyeballs were removed and processed for histopathology. Isolated retinal tissues were fixed in $4 \%$ paraformaldehyde, followed by cryoprotection in $30 \%$ sucrose, dehydration in increasing concentrations of alcohol, paraffin embedding, sectioning (thickness, $2 \mu \mathrm{m}$ ), and staining with hematoxylin and eosin for microscopic observation $(26,27)$.
Terminal deoxynucleotidyl-transferase-mediated dUTP nick end labeling (TUNEL) assays. Paraffin-embedded sections of the retinal layers of the eye specimens were deparaffinized and dried in a $60^{\circ} \mathrm{C}$ oven for $1 \mathrm{~h}$. Sections were deparaffinized in two xylene washes and hydrated with an ethanol gradient (100, 95, 90, 80 and 75\%; $5 \mathrm{~min} /$ concentration). Sections were immersed in three 5 min PBS washes. Each section was treated with $100 \mu \mathrm{l}$ freshly-prepared Proteinase $\mathrm{K}$ solution $(98 \mu \mathrm{l} \mathrm{PBS}+2 \mu \mathrm{l} 50 \mathrm{X}$ Proteinase $\mathrm{K})$ at $37^{\circ} \mathrm{C}$ for $30 \mathrm{~min}$, and subsequently washed three times for 5 min with PBS. Sections were treated with $50 \mu 1$ TUNEL solution $(2 \mu 1 \mathrm{TdT}+48 \mu 1$ fluorescent labeling solution) to detect apoptosis (In Situ Cell Death Detection kit; Roche Diagnostics GmbH, Mannheim, Germany) for $1 \mathrm{~h}$ at $37^{\circ} \mathrm{C}$ in the dark. TUNEL signals were 

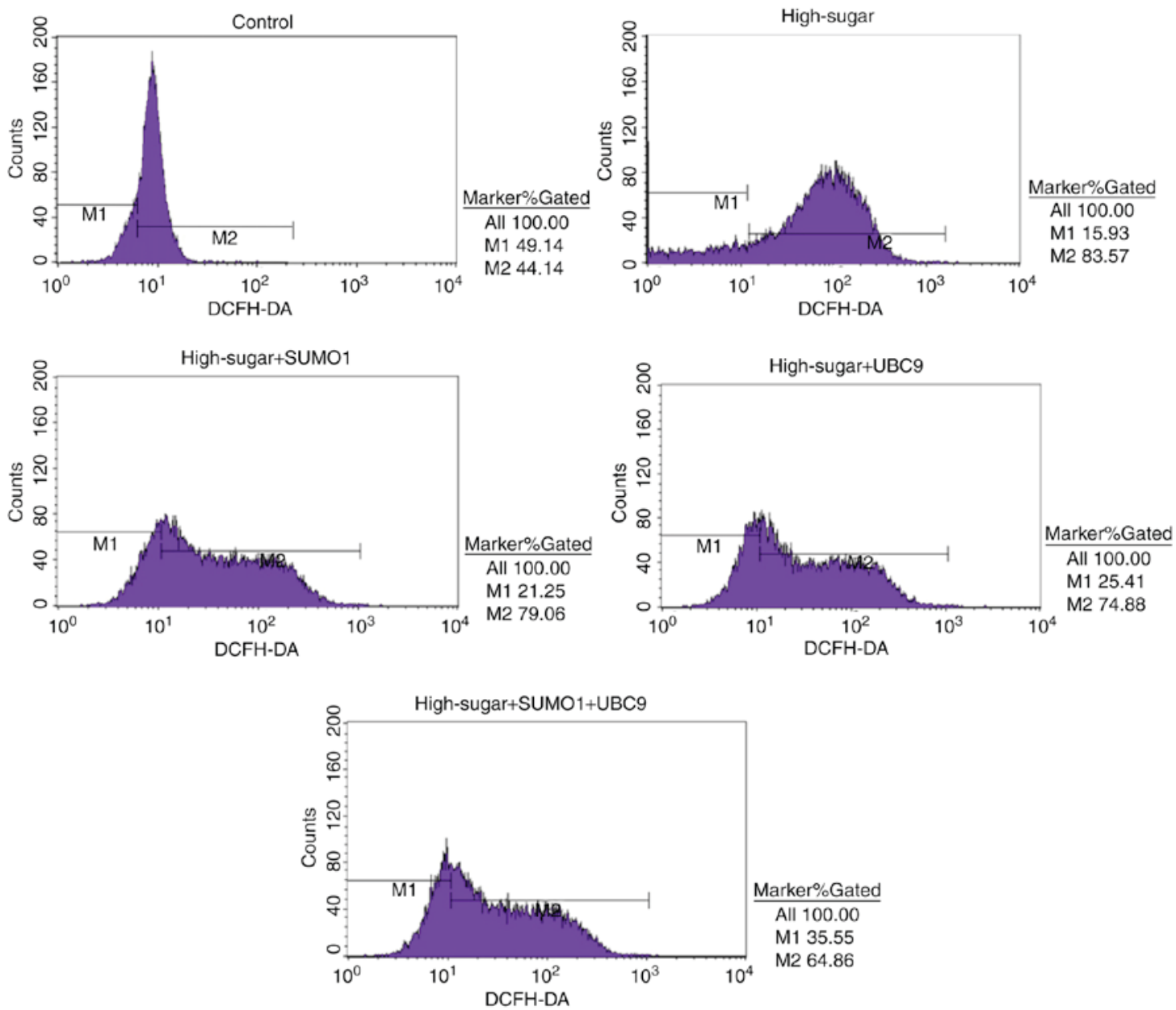

Figure 4. Intracellular reactive oxygen species quantities were assessed using DCFH-DA staining by flow cytometry at $488 \mathrm{~nm}$ excitation and $525 \mathrm{~nm}$ emission. DCFH-DA, dichlorodihydrofluorescein diacetate; SUMO1, small ubiquitin-like modifier 1; UBC9, ubiquitin conjugating enzyme E2 I.

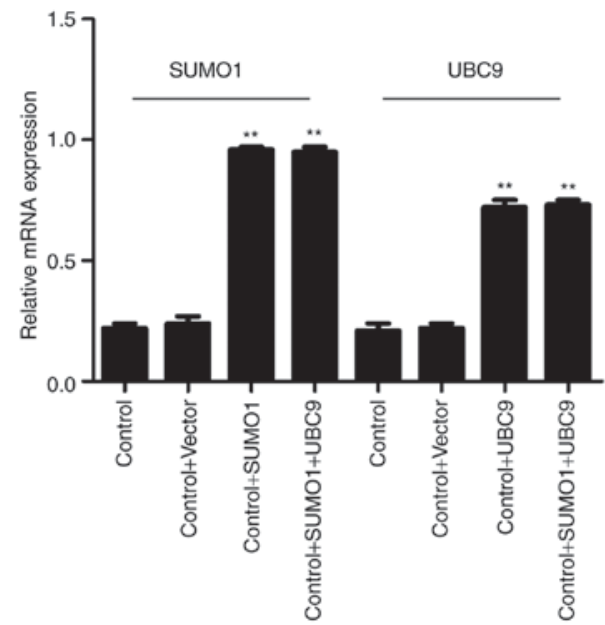

Figure 5. Relative mRNA expression of $S U M O 1$ and $U B C 9$, analyzed by reverse transcription-quantitative polymerase chain reaction in rat retinal tissues following infections with lentiviruses expressing SUMO1 and/or $U B C 9$. $\beta$-actin was used as the reference/housekeeping gene. ${ }^{* *} \mathrm{P}<0.01$ vs. controls. SUMO1, small ubiquitin-like modifier $1 ; U B C 9$, ubiquitin conjugating enzyme E2 I.

measured using a fluorescence microscope (20 fields, Olympus IX81; Olympus Corporation, Tokyo, Japan).
Statistical analyses. All experiments were performed three times independently in triplicate. The data are presented as the mean \pm standard deviation. Comparisons between two groups were analyzed using two-tailed Student's t-tests; data across groups were analyzed using one-way analysis of variance tests followed by the Least Significant Difference post hoc test. Statistical analyses were performed using SPSS 17.0 software (SPSS, Inc., Chicago, IL, USA). P $<0.05$ was considered to indicate a statistically significant difference.

\section{Results}

Overexpression of SUMO1 and/or UBC9 alters ROS and apoptosis levels following exposure to high-sugar conditions. In order to evaluate the individual effects of SUMO1 and UBC9 overexpression and their combined overexpression in high-sugar conditions, lentiviral transfections were used to introduce the SUMO1, UBC9 and SUMO1+UBC9 genes into Nox1-expressing HRECs. Post-transfection, the relative mRNA and protein expression levels of SUMO1 and UBC9 were upregulated significantly in all groups compared with the controls $(\mathrm{P}<0.01$; Fig. $1 \mathrm{~A}$ and $\mathrm{B})$. Following exposure to high sugar, the mRNA expression levels of SUMO1, UBC9 and Nox1 were analyzed; expression levels were increased 1.75-2.22 $(\mathrm{P}<0.05-\mathrm{P}<0.01), 2.04-2.93(\mathrm{P}<0.05-\mathrm{P}<0.01)$ and 

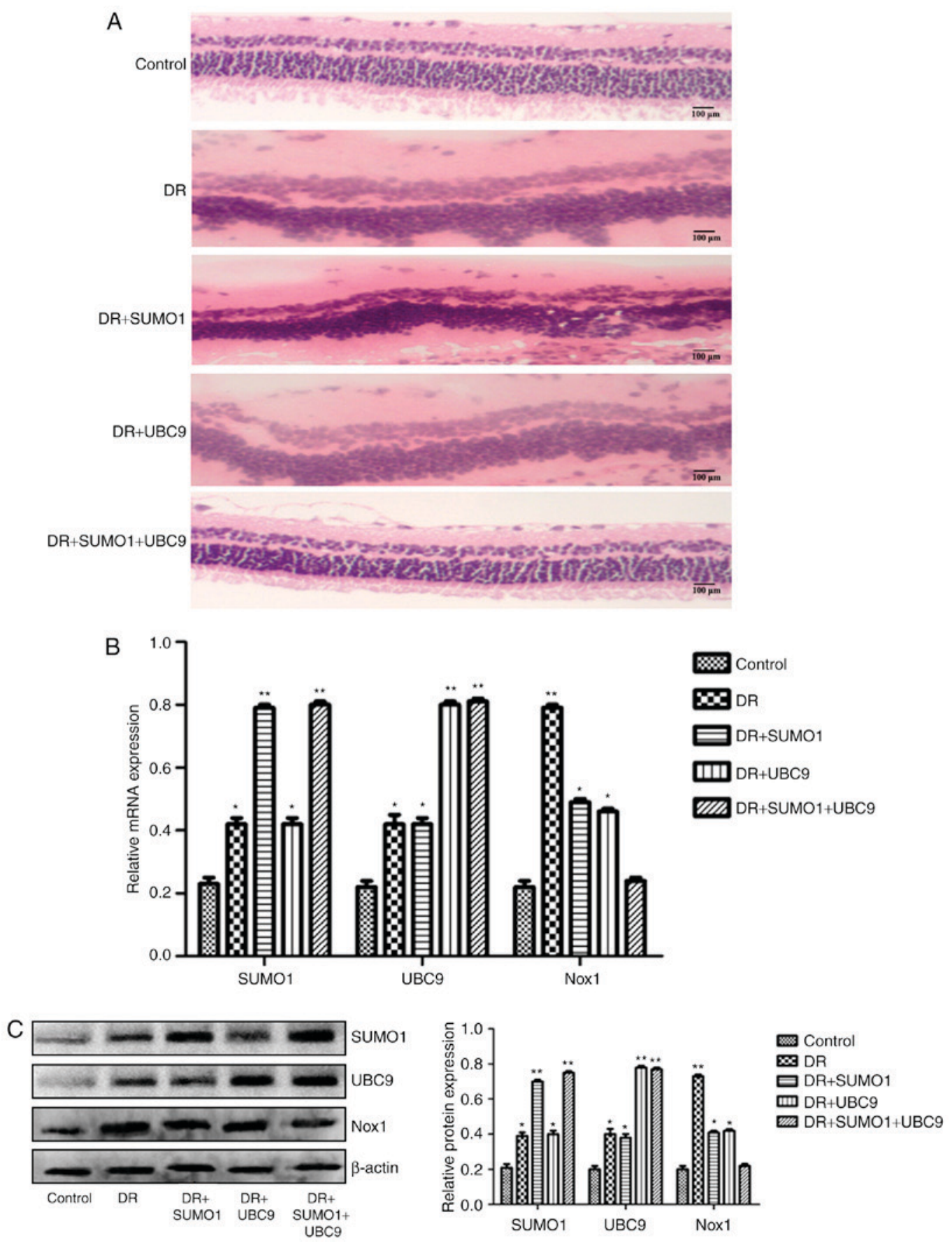

Figure 6. Effect of lentiviral transfection of SUMO1 and UBC9 on retinal tissue pathology and mRNA and protein levels of SUMO1, UBC9 and Nox1 in an established DR model. (A) Pathological evaluation of retinal tissues. Relative (B) mRNA and (C) protein expressions of SUMO1, UBC9 and Noxl in an established rat DR model across five treatment groups: Healthy controls (control), DR affected animals (DR), DR animals infected with $S U M O 1$ expressing lentiviruses (DR + SUMO1), DR animals infected with UBC9 lentiviruses (DR + UBC9), and DR animals infected with both SUMO1 and UBC9 lentiviruses (DR + SUMO1 + UBC9). ${ }^{*} \mathrm{P}<0.05,{ }^{* *} \mathrm{P}<0.01$ vs. control. SUMO1, small ubiquitin-like modifier 1; UBC9, ubiquitin conjugating enzyme E2 I; Noxl, NADPH oxidase 1; DR, diabetic retinopathy.

1.03-2.77 $(\mathrm{P}<0.05-\mathrm{P}<0.01)$ fold, respectively, across the different treatment groups versus the control (Fig. 1C). The survival rates of high sugar-exposed transfected cells were quantified using Hoechst 33258 staining. Morphologically, apoptotic cells were round, with condensed nuclei and high fluorescence levels (Fig. 2A). Cell death rates decreased in high sugar-exposed cell lines transfected with either SUMO1 $(\mathrm{P}<0.001)$ or UBC9 $(\mathrm{P}<0.001)$. However, high sugar-exposed cells that were co-infected with SUMO1 and UBC9 exhibited no significant alterations in cell death rates compared with controls (Fig. 2B). In addition, apoptosis rates and ROS production rates were assessed in the models using annexin $\mathrm{V} /$ propidium iodide staining and DCFH-DA conversion assays, respectively. In high-glucose treated HRECs, the apoptosis rates were $\sim 51.6 \%(\mathrm{P}<0.01)$. However, the introduction of either SUMO1 or UBC9 significantly decreased the apoptosis rate to $38.7 \%(\mathrm{P}<0.01)$ and $36.5 \%(\mathrm{P}<0.01)$, respectively. In high-glucose treated HRECs co-transfected with SUMO1 and UBC9, apoptosis levels were comparable to controls (Fig. 3). Similarly, infection with SUMO1 or UBC9 decreased ROS 


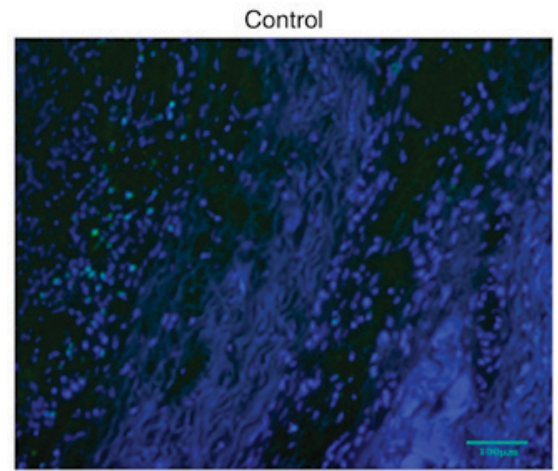

DR+SUMO1

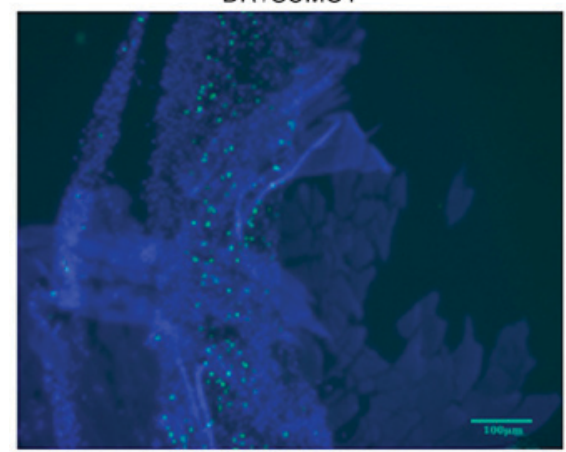

DR

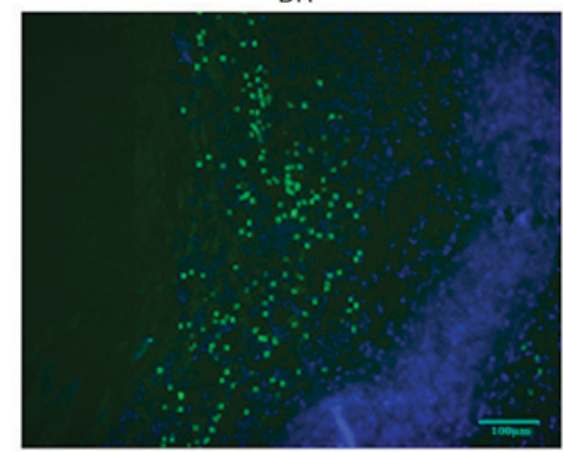

DR+UBC9

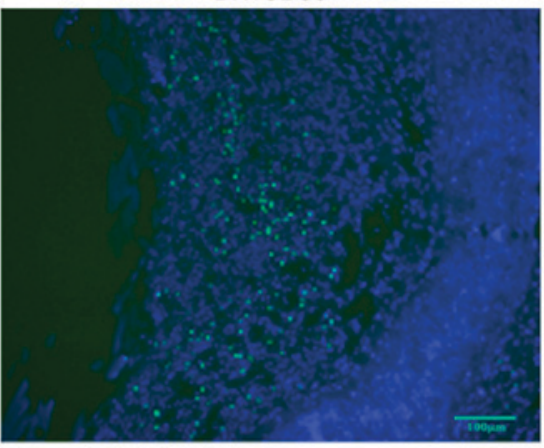

DR+SUMO1+UBC9

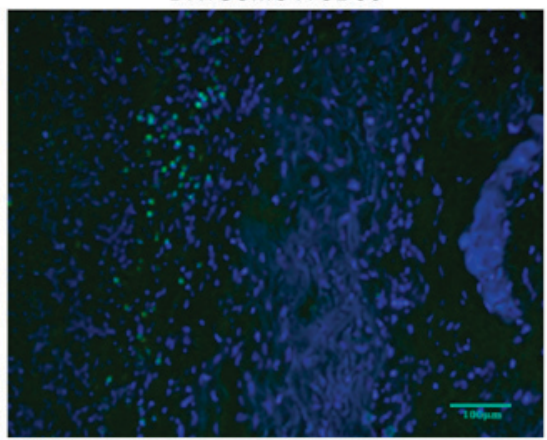

Figure 7. Representative images of apoptotic events were visualized following TUNEL assays in retinal tissues from the DR models. DR, diabetic retinopathy; SUMO1, small ubiquitin-like modifier 1; UBC9, ubiquitin conjugating enzyme E2 I.

generation in high sugar-exposure conditions, while co-transfection with SUMO1 and UBC9 further decreased ROS levels in these conditions (Fig. 4).

Infection with SUMO1-and/or UBC9-expressing lentiviruses alters the onset of $D R$. A rat model was established to assess the roles of SUMO1, UBC9 and Nox1 in the regulation of DR. Infections of the animals with SUMO1, UBC9 or SUMO1 + UBC9 increased the mRNA expression levels of SUMO1 and/or UBC9 compared with controls $(\mathrm{P}<0.01$; Fig. 5). In retinal sections from DR rats, morphological alterations were observed in the inner nuclear layers (INL) and outer nuclear layers (ONL) between the five groups. Compared with the healthy controls, the staining patterns were uneven, cellular morphologies were incomplete, and the presence of vacuoles was increased in the INL and ONL in the DR animals. However, following infection with SUMO1 and UBC9, the structures of the retinal layers were clear and the incidences of injured tissues were decreased (Fig. 6A). Post-infection mRNA expression levels of SUMO1, UBC9 and Nox1 rose by 1.83-3.48 $(\mathrm{P}<0.05-\mathrm{P}<0.01), 1.91-3.68(\mathrm{P}<0.05-\mathrm{P}<0.01)$ and 1.09-3.68 $(\mathrm{P}<0.05-\mathrm{P}<0.01)$ fold, respectively, across the different treatment groups versus the respective controls (Fig. 6B). Immunofluorescence imaging and relative quantitation of the western blotting assays confirmed the results of the gene expression analyses (Fig. 6C). TUNEL assays were employed to detect the effects of SUMO1 and UBC9 overexpression on apoptosis in DR models. Compared with the DR group, Nox1-induced apoptosis was inhibited; the number of cells with a fluorescent signal above background decreased in the presence of SUMO1 or UBC9 overexpression. Notably, apoptosis was more effectively inhibited when SUMO1 or UBC9 was transfected individually, compared with SUMO1 and UBC9 co-transfection (Fig. 7).

\section{Discussion}

The results of the present study demonstrated that overexpression of either SUMO1 or the SUMO-specific conjugating enzyme, UBC9, decreased Nox1 expression and Nox1-mediated ROS production. Additionally, Nox1 and ROS inhibition was potentiated by the combined overexpression of 
SUMO1 and UBC9 following exposure to high-sugar media. In addition to inhibiting Nox1 expression levels and ROS production, overexpression of SUMO1 or UBC9 decreased apoptosis rates in retinal endothelial cells and relived DR symptoms in animal models. The results of the present study suggested that SUMO1 may be an endogenous suppressor of Nox1-induced ROS production in DR model systems.

SUMOylation regulates a series of biological activities, including gene product production, intracellular protein localization, protein-protein interactions and signal transduction (12). SUMOylation may be initiated by stressors, including heat, osmosis and ROS $(14,27)$, and exhibits increased activity in cases of diabetes (18). SUMOylation is considered to be cytoprotective. However, not all SUMOylation activities promote cell survival. In the present study, Nox1 expression levels and ROS were regulated by SUMO1. This likely functioned to limit further cellular damage by decreasing ROS, exhibiting the protective nature of SUMOylation. Previous studies have demonstrated that the transcriptional activities of extracellular-signal-regulated kinase-5 was downregulated by increased SUMOylation, resulting in worsened cardiac function following myocardial ischemia (28). Additionally, ROS-induced tissue transglutaminase SUMOylation promoted inflammation (29).

It is well-understood that SUMO conjugates with its substrates through activation of the (E1) enzyme [SUMO activating enzyme subunit (SAE)1/SAE2] and a conjugating (E2) enzyme (UBC9). This complex efficiently transfers SUMO to selected targets by binding to SUMO acceptor sites (18). Therefore, UBC9-mediated intracellular transport is dependent on SUMOylation. There is also evidence that UBC9-mediated intracellular transport may occur independently of SUMOylation. UBC9 participated in visual system homeobox 1 (Vsx1) transport from the nucleus to the cytoplasm; Vsx 1 is a homeobox transcription factor which is dynamically expressed during zebrafish development (30). UBC9 has additionally been demonstrated to transport DNA repair protein RAD51 homolog 1, a highly-conserved protein in all eukaryotes, out of the nucleus (31). In the present study, transfection of SUMO1 alone did not increase UBC9 mRNA or protein expression in HREC cells (Fig. 1) or the DR model (Fig. 6). The results of the present study suggested that Nox1-mediated ROS production was regulated by UBC9-mediated intracellular transport that was dependent on SUMOylation.

Nox1 protein has subcellular localization in the plasma membrane, the endoplasmic reticulum, caveolae and endosomes (16). In previous studies, it was observed that SUMO1 was able to alter the activity of Nox family members indirectly, and that Nox 2 was considered to be a target protein of SUMO1 $(14,15)$. However, these previous studies did not report evidence of direct binding of SUMO1 with Nox2 or Nox5. Given that no Nox1-specific antibodies are currently available, it has been difficult to determine whether Nox1 localization regulates its function. As previously described, Nox1 was the predominant isoform in retinal ganglion cells, and the present study demonstrated that combined SUMO1 and UBC9 overexpression significantly inhibited Nox 1 . Therefore, the results of the present study suggested that Nox1 localization was associated with its function. Future studies may focus on elucidating whether SUMO1 directly conjugates with Nox1 and whether Nox1 is a SUMO1 substrate.

In conclusion, the role of inhibition of SUMO1 overexpression in Nox1 activity, ROS production and apoptosis may be potentiated by co-overexpression with UBC9 in vitro and in vivo. The results suggested that endogenous SUMO1 may suppress Nox1-controlled ROS production in DR via SUMOylation-dependent UBC9-mediated intracellular transport. Further studies are required to elucidate the mechanism underlying SUMO1 and Nox1 activity, and to demonstrate the direct conjugation of SUMO1 and Nox1.

\section{Acknowledgements}

The present study was supported by a Project for Department of Science and Technology grant from the Jiangxi Province (grant no. 2014BBG70043).

\section{References}

1. Zhang X, Saaddine JB, Chou CF, Cotch MF, Cheng YJ, Geiss LS, Gregg EW, Albright AL, Klein BE and Klein R: Prevalence of diabetic retinopathy in the United States, 2005-2008. JAMA 304: 649-656, 2010.

2. Fong DS, Aiello L, Gardner TW, King GL, Blankenship G, Cavallerano JD, Ferris FL III and Klein R; American Diabetes Association: Retinopathy in diabetes. Diabetes Care 27 (Suppl 1): S84-S87, 2004

3. Klein R, Klein BEK, Moss SE, Davis MD and DeMets DL: The wisconsin epidemiologic study of diabetic retinopathy: II. Prevalence and risk of diabetic retinopathy when age at diagnosis is less than 30 years. JAMA Ophthalmol 102: 520-526, 1984.

4. Behl T, Kaur I and Kotwani A: Implication of oxidative stress in progression of diabetic retinopathy. Surv Ophthalmol 61: 187-196, 2016.

5. Safi SZ, Qvist R, Kumar S, Batumalaie K and Ismail IS: Molecular mechanisms of diabetic retinopathy, general preventive strategies, and novel therapeutic targets. Biomed Res Int 2014: 801269, 2014.

6. Schippers JH, Nguyen HM, Lu D, Schmidt R and Mueller-Roeber B: ROS homeostasis during development: An evolutionary conserved strategy. Cell Mol Life Sci 69: 3245-3257, 2012.

7. Wilkinson-Berka JL, Rana I, Armani R and Agrotis A: Reactive oxygen species, Nox and angiotensin II in angiogenesis: Implications for retinopathy. Clin Sci. (Lond.) 124: 597-615, 2013.

8. Bondeva $\mathrm{T}$ and Wolf $\mathrm{G}$ : Reactive oxygen species in diabetic nephropathy: Friend or foe? Nephrol Dial Transplant 29: 1998-2003, 2014.

9. Brandes RP, Weissmann N and Schröder K: Nox family NADPH oxidases: Molecular mechanisms of activation. Free Radic Biol Med 76: 208-226, 2014.

10. Dvoriantchikova G, Grant J, Santos AR, Hernandez E and Ivanov D: Neuronal NAD $(\mathrm{P}) \mathrm{H}$ oxidases contribute to ROS production and mediate RGC death after ischemia. Invest Ophthalmol Vis Sci 53: 2823-2830, 2012.

11. Gill G: SUMO and ubiquitin in the nucleus: Different functions, similar mechanisms? Genes Dev 18: 2046-2059, 2004.

12. Johnson ES: Protein modification by SUMO. Annu Rev Biochem 73: 355-382, 2004.

13. Hwang KW, Won TJ, Kim H, Chun HJ, Chun T and Park Y: Characterization of the regulatory roles of the SUMO. Diabetes Metab Res Rev 27: 854-861, 2011.

14. Pandey D, Chen F, Patel A, Wang CY, Dimitropoulou C, Patel VS, Rudic RD, Stepp DW and Fulton DJ: SUMO1 negatively regulates reactive oxygen species production from NADPH oxidases. Arterioscler Thromb Vasc Biol 31: 1634-1642, 2011.

15. Kim HJ, Yun J, Lee J, Hong H, Jeong J, Kim E, Bae YS and Lee KJ: SUMO1 attenuates stress-induced ROS generation by inhibiting NADPH oxidase 2. Biochem Biophys Res Commun 410: 555-562, 2011.

16. Gimenez M, Schickling BM, Lopes LR and Miller FJ Jr: Nox1 in cardiovascular diseases: Regulation and pathophysiology. Clin Sci (Lond) 130: 151-165, 2016. 
17. Chhunchha B, Fatma N, Kubo E and Singh DP: Aberrant sumoylation signaling evoked by reactive oxygen species impairs protective function of Prdx6 by destabilization and repression of its transcription. FEBS J 281: 3357-3381, 2014.

18. Li M, Guo D, Isales CM, Eizirik DL, Atkinson M, She JX and Wang CY: SUMO wrestling with type 1 diabetes. J Mol Med (Berl) 83: 504-513, 2005.

19. Arden GB and Sivaprasad S: Hypoxia and oxidative stress in the causation of diabetic retinopathy. Current Diabetes Rev 7: 291-304, 2011.

20. Liu GD, Xu C, Feng L and Wang F: The augmentation of O-GlcNAcylation reduces glyoxal-induced cell injury by attenuating oxidative stress in human retinal microvascular endothelial cells. Int J Mol Med 36: 1019-1027, 2015.

21. Banskota S, Regmi SC and Kim JA: NOX1 to NOX2 switch deactivates AMPK and induces invasive phenotype in colon cancer cells through overexpression of MMP-7. Mol Cancer 14: 123, 2015.

22. Valente AJ, El Jamali A, Epperson TK, Gamez MJ, Pearson DW and Clark RA: NOX1 NADPH oxidase regulation by the NOXA1 SH3 domain. Free Radic Biol Med 43: 384-396, 2007.

23. Livak KJ and Schmittgen TD: Analysis of relative gene expression data using real-time quantitative PCR and the 2(-Delta Delta C(T)) method. Methods 25: 402-408, 2001.

24. Li Z, Ma L, Chen X, Li Y, Li S, Zhang J and Lu L: Glycogen synthase kinase-3: A key kinase in retinal neuron apoptosis in early diabetic retinopathy. Chin Med J (Engl.) 127: 3464-3470, 2014.

25. Jia J, Zhang X, Hu YS, Wu Y, Wang QZ, Li NN, Guo QC and Dong XC: Evaluation of in vivo antioxidant activities of Ganoderma lucidum, polysaccharides in STZ-diabetic rats. Food Chem 115: 32-36, 2009

26. Gong CY, Lu B, Hu QW and Ji LL: Streptozotocin induced diabetic retinopathy in rat and the expression of vascular endothelial growth factor and its receptor. Int J Ophthalmol 6 : $573-577,2013$
27. Xiao X, Cai J, Xu J, Wang R, Cai J, Liu Y, Xu W, Sun X and Li R: Protective effects of hydrogen saline on diabetic retinopathy in a streptozotocin-induced diabetic rat model. J Ocul Pharmacol Ther 28: 76-82, 2012.

28. Hong Y, Rogers R, Matunis MJ, Mayhew CN, Goodson ML, Park-Sarge OK and Sarge KD: Regulation of heat shock transcription factor 1 by stress-induced SUMO-1 modification. J Biol Chem 276: 40263-40267, 2001.

29. Shishido T, Woo CH, Ding B, McClain C, Molina CA, Yan C, Yang $\mathrm{J}$ and Abe J: Effects of MEK 5/ERK5 association on small ubiquitin-related modification of ERK5: Implications for diabetic ventricular dysfunction after myocardial infarction. Circ Res 102: 1416-1425, 2008

30. Luciani A, Villella VR, Vasaturo A, Giardino I, Raia V, Pettoello-Mantovani M, D'Apolito M, Guido S, Leal T, Quaratino S and Maiuri L: SUMOylation of tissue transglutaminase as link between oxidative stress and inflammation. J Immunol 183: 2775-2784, 2009.

31. Kurtzman AL and Schechter N: Ubc9 interacts with a nuclear localization signal and mediates nuclear localization of the paired-like homeobox protein Vsx-1 independent of SUMO-1 modification. Proc Natl Acad Sci USA 98: 5602-5607, 2001.

This work is licensed under a Creative Commons Attribution-NonCommercial-NoDerivatives 4.0 International (CC BY-NC-ND 4.0) License. 